\title{
Manifestaciones intraoculares del cáncer sistémico
}

\author{
J.M. ${ }^{a}$ Caminal-Mitjana
}

\begin{abstract}
INTRAOCULAR FEATURES OF SYSTEMIC CANCER
Summary. Systemic cancer may present intraocular features in three forms: as a sign of the presence of lymphoreticular tumors, of solid tumors or as a paraneoplastic syndrome. [REV NEUROL 2000; 31: 1259-61] [http://www.revneurol.com/3112/ j121259.pdf]

Key words. Intraocular manifestations. Lymphoproliferative tumors. Paraneoplastic syndrome. Systemic cancer.
\end{abstract}

Podemos resumir las manifestaciones intraoculares del cáncer sistémico en tres grandes apartados:

1. Manifestaciones de tumores linfoproliferativos

2. Manifestaciones de tumores sólidos

3. Efectos a distancia del cáncer. Síndromes paraneoplásicos.

\section{MANIFESTACIONESDE TUMORES LINFOPROLIFERATIVOS}

Las leucemias son procesos malignos de la serie blanca sanguínea, del tipo mieloide o linfoide, y cada una de ellas puede ser aguda o crónica. Las leucemias y los tumores linfoides pueden afectar cualquier parte del ojo y de los anejos oculares, aunque los encontramos con mayor frecuencia en la órbita y conjuntiva, y de manera mucho menos frecuente en las estructuras intraoculares

Lainfiltración leucémica de las estructuras intraoculares sólo ocurre en el $3 \%$ de pacientes; en cambio, en el $40-50 \%$ de los casos observamos alteraciones asociadas a los cambios hematológicos que encontramos en la mayoría de pacientes [1]. Los infiltrados iridianos se caracterizan por un engrosamiento blanquecino difuso, a menudo con pequeños nódulos en el margen pupilar, y puede asociarse a un pseudohipopión y glaucoma. Los infiltrados de células leucémicas en el segmento posteriorpodemos hallarlosen la retina, el espacio subretiniano y en la coroides; presentan un aspecto amarillento y debemos diferenciarlos de infecciones virales o fúngicas oportunistas, especialmente en pacientes inmunosuprimidos.La afectación retiniana y vítrea secundaria al trastornohematológico(anemia, trombopenia, hiperviscosidad) secaracteriza por hemorragias intrarretinianas, manchas algodonosas, hemorragias con el centro blanquecino, oclusiones de la vena central de la retina y hemorragias vítreas.

En la mayoría de las ocasione, el diagnóstico se realiza por la presenciadeenfermedad previaconocida, peroen aquellos casos en que no existe una leucemia diagnosticada es imperativa la realización de un examen físico, un hemograma completo y un estudio de médulaósea. Labiopsia de los tejidos oculares afectados (aspiración del humor acuoso, punción-aspiración con aguja

Recibido: 31.03.00. Aceptado: 27.04.00.

Servicio de Oftalmología. Hospital Prínceps d'Espanya. Ciutat Sanitàriai Universitària de Bellvitge. Universitat de Barcelona. Barcelona, España.

Correspondencia: Dr. Josep M. ${ }^{a}$ Caminal Mitjana. Servei d'Oftalmologia. Hospital Prínceps d'Espanya. Ciutat Sanitària i Universitària de Bellvitge. Universitat de Barcelona. Feixa Llarga, s/n. E-08907 L'Hospitalet de Llobregat, Barcelona.

(C) 2000, REVISTA DENEUROLOGÍA fina transvítrea, obiopsia con el vitreotomo) es el mejor método diagnóstico del procesoleucémico.

Las opciones de tratamiento son la radioterapia externa obien laquimioterapia. Enelcaso de la radioterapia se emplean dosis de 2.000-3.000 cGy, aplicadas en dosis fraccionadas de $200 \mathrm{cGy}$.

El linfoma no hodgkiniano $(\mathrm{LNH})$ tiene dos presentaciones clínicas: elLNH sistémico y el LNH del sistema nervioso central (LNH-SNC), que también se conoce como linfoma del SNC primario y se desarrolla a partir del cerebro, médula espinal, leptomeninges odelojo[2].Las manifestaciones oculares pueden ocurrir en las dos entidades, pero con mayor frecuencia aparecen con el LNH-SNC.

El linfoma primario intraocular(linfoma de células grandes) es una entidad rara que forma parte del espectro clínico del LNH-SNC. A menudo, se presenta como una uveítis crónica en pacientes de edad avanzada. En general, es de inicio unilateral, pero frecuentemente con afectación bilateral. El proceso se presenta como una infiltración celular difusa del vítreo y como acúmulos de células linfomatosas (linfocitos tipo B) a nivel subretiniano. Estas últimas lesiones clínicamente tienen un aspecto amarillento y se presentan con un patrón geográfico, rodeadas de nódulos satélites; también puede afectarse la coroides y el nervioóptico.

Eldiagnóstico de linfomaintraocular primario, en ausencia de afectación cerebral, a menudo requiere de una biopsia vítrea con posterioranálisiscitológico, asícomoladeterminacióndenivelesde interleucina-10[3,4].Enaquellospacientesconlesiones subretinianas ocoroideas puedeserútillapunción-aspiración conagujafina.

El tratamientoconsiste generalmente en lairradiación externa de ambos globos en aquellos casos con afectación ocular; en el caso de implicación del SNC, entonces se utiliza una pauta de radioterapiaasociadaaquimioterapiasistémicae intratecal.

\section{MANIFESTACIONES DE TUMORES SÓLIDOS}

Las metástasis son latumoraciónintraocularmás frecuente. Aproximadamente un 5\% de los pacientes con cáncer sistémico presentan metástasis coroideas, aunque la incidencia de lesiones metastásicas es superior en autopsias que en estudios clínicos. Laedad media de presentación está en los 60 años. La localización más frecuente del cáncer primario es en la mama [5], seguido por el pulmón. Otros tumores capaces de metastatizar en la úvea son el carcinoma de riñón y el carcinoma de próstata, así como el melanoma cutáneo. Los tumores gastrointestinales, que representan el $15 \%$ de los cánceres en eladulto, raramente metastatizan en elojo.

La mayoría de tumores que metastatizan en el ojo son carcinomas, quelleganalaúveamediante diseminaciónhematógena. De 
manerapredominante, se afectala porción posterior de lacoroides, probablemente debidoa que es lazonamejor vascularizada, consus 20 arterias ciliares posteriores cortas[6]. Aproximadamenteel40\% de las lesiones metastásicas afectan la coroides submaculary, en general, los cuadrantes temporales están más afectados que los nasales. Raramente se afecta el iris y el cuerpo ciliar, y cuando se afecta el primero suele ser por un carcinoma de pulmón.

Las metástasis de iris tienen un aspecto nodular y a menudo infiltrativo, con siembras sobre la superficie. Típicamente tienen un aspecto rosado-grisáceo o blanco-grisáceo y pueden debutar como un cuadro de uveítis o de glaucoma. Estas lesiones pueden ser dolorosas como consecuencia de la invasión de los nervios ciliares.

Las lesiones metastásicas coroideas generalmente son amarillo-grisáceas oblanco-rosadas. A menudo son multicéntricas y bilaterales, de base amplia y poca elevación, con bordes irregulares y pueden presentar acúmulos pigmentarios en su superficie. En la mayoría de pacientes se evidencia un amplio desprendimiento seroso de retina. En general, las metástasis coroideas se presentan como pérdida de visión [7], probablemente debido al hecho de que la mayoría de las lesiones afectan al polo posterior y dan clínica con mayor precocidad; también pueden presentarse comodefectos campimétricos y miiodesopsias.

La angiografía fluoresceínica menudo muestra hipofluorescencia en la fase arterial y venosa precoz, y una progresiva hiperfluorescencia en las fases siguientes, a diferencia del hemangioma y melanomadecoroides. Enlaexploración ecográficaen modoA, generalmente presenta una amplitud de reflectividad interna de moderadaaelevada, adiferenciadel melanomadeúveaquemuestra unabajareflectividadinterna [8].Enlaecografía en modo-B, de forma habitual encontramos una masa tumoral coroidea con un patrón acústico de moderado a alto, sin excavación coroidea, ni sombra orbitaria. Con cierta frecuencia encontramos lesiones multifocales.

La topografía computarizada no es útil en el diagnóstico diferencial de las tumoraciones coroideas. En cambio, la resonancia magnéticanuclearpermite en determinados casos diferenciarestas lesiones del melanoma maligno de úvea. Además, esta técnica posibilita la exploración simultánea del SNC en busca de otras lesiones metastásicas.

La punción con aguja fina es una técnica relativamente segura, aunque invasiva y con riesgos potenciales, reservada para aquellos casos en que existauna dudadiagnóstica razonable, a pesarde las exploraciones complementarias menos cruentas [9].

Siempreque sospechemos que unalesión pueda sermetastásica, debemos realizar una exploración física completa, así como unaradiografía de tórax, analítica general coninclusión del antígeno carcinoembrionario, y del antígeno prostático específico [10].

Eldiagnóstico de una metástasis coroidea es un signode mal pronóstico, porque indica una amplia diseminación de la enfermedadmetastásica, con una supervivencia de 6-12 meses después de la presentación oftalmológica [11]. Los carcinomas de pulmón, próstata y renal tienden a metastatizar más frecuentemente, por lo que es habitual diagnosticar el tumor primario, tras diagnosticar la lesión coroidea. En cambio, el carcinoma de mama y el melanoma cutáneo tienden a metastatizar tardíamente, por lo que la lesión coroidea suele diagnosticarse después del tumor primario.

A pesar del mal pronóstico para los pacientes con metástasis uveales, debeinstaurarsetratamientoconprontitud,puestoqueestas lesiones pueden originar ceguera (en casos bilaterales o en ojos únicos)obien dolor.Lasopciones detratamientoson:radioterapia, quimioterapia(y terapiahormonal), resección quirúrgica(en lesiones uveales anteriores), enucleación y observación. Laelección del tratamiento depende de varios factores: el efecto del tumoren la función visual del ojo, el tamaño del tumor, el estado delojocontralateral, el estadoy expectativa de vida del paciente, y siel proceso sistémicoestásiendotratadoconquimioterapia[12].

En la mayoría de pacientes se utiliza radioterapia externa convencional, con una dosis que varía entre 2.500-5.000 cGy a través de un campo orbitariolateral, en fracciones de 200 cGy, en un período de tres a cuatro semanas. En el caso de lesiones coroideas únicas, sin otra afectación sistémica, puede plantearse la utilización de placas episclerales radioactivas (braquiterapia).

\section{EFECTOS A DISTANCIA DEL CÁNCER. SÍNDROMES PARANEOPLÁSICOS}

La retinopatía asociada al cáncer es un proceso paraneoplásico raro que origina pérdida visual, producida por una disfunción retiniana no asociada a invasión directa tumoral, toxicidad por quimioterapia, deficiencias nutricionales o infecciones oportunistas. Con mayor frecuencia, se asocia al carcinoma de pulmón de pequeñas células (oat-cell), aunque también se ha descritocon otros cánceres, como el carcinoma de cérvix, sarcoma uterino o carcinoma de mama.

Generalmente, estos pacientes se presentan con una progresiva pérdida bilateral de visión, ceguera nocturna y alteraciones en el campo visual (escotomas, visión en túnel). Algunos pacientes refieren nictalopía y otros deslumbramiento y fotosensibilidad; posiblemente ello refleja la diferente afectación de bastones en unos individuos o de conos en otros.

En la exploración podemos encontrar iridociclitis o vitritis leve, estrechamiento de las arteriolas, alteraciones del epitelio pigmentario de la retina y palidez del disco óptico. Los defectos campimétricosquegeneralmente encontramos sonescotomasen media periferia, escotomas en anillo o defectos centrales. Elelectrorretinograma es de gran utilidad en el diagnóstico de la retinopatía asociadaalcáncer, puestoque en la mayoría de los pacientes encontramos una reducción o un aplanamiento de sus amplitudes. Tanto se afecta el sistema de los conos como el de los bastones.

En 1987, Thirkill et al [13] publicaron la determinación en sangre periférica de anticuerpos antirretinianos, que se unían aun antígenodepesomolecular23.000 Daltons(antígenodelaretinopatía asociada al cáncer) [13]; ello evidenció que este es un proceso de autoinmunidad, en el que existe una reacción cruzada entre las células tumorales y las células retinianas.

Se han publicado resultados positivos en algunos pacientes mediante tratamientoinmunosupresorcon corticosteroides en dosis de 60 a $80 \mathrm{mg}$ [14]. Parece que el control de la neoplasia primaria noinfluye en la evolución del proceso visual.

Además de la retinopatía asociada al cáncer se han descrito otros síndromes paraneoplásicos, entre los que encontramos: retinopatía asociada a melanoma (variante de la primera asociada únicamente al melanoma maligno cutáneo), la proliferación melanocítica uveal difusa y la neuropatíaóptica paraneoplásica.

La proliferación melanocítica uveal difusa es una retinopatía paraneoplásica que se ha asociado a neoplasias del tracto reproductivo femenino (carcinoma de ovario). Típicamente los pacientes presentan la proliferación bilateral de numerosas lesiones subretinianas de color amarillo-naranja o pigmentadas, que se corresponde anatomopatológicamente a una proliferación benigna de melanocitos. Se desconoce la patogenia del proceso [15]. 


\section{BIBLIOGRAFÍA}

1. Schachat AP, Markovitz JA, Guyer DR, et al. Ophthalmic manifestations of leukemia. Arch Ophthalmol 1989; 107: 697-700.

2. Freilich RJ, DeAngelis LM. Primary central nervous system lymphoma. Neurol Clin 1995; 13: 901-14.

3. Davis JL, Soloman D, Nussenblatt RB, et al. Immunocytochemical staining of vitreous cells. Ophthalmology 1992; 99: 250-6.

4. Chan CC, Whitcup SM, Solomon D, Nussenblatt RB. Interleukin-10 in the vitreous of patients with primary intraocular lymphoma. Am J Ophthalmol 1995; 120: 671-3.

5. Reese AB. Metastatic tumors of the eye and adnexa. In Reese AB, ed. Tumors of the eye. 3 ed. Hagerstown: Harper \& Row; 1976. p. 423-31

6. Freedman MI, Folk JC. Metastasic tumors to the eye and orbit: patient survival and clinical characteristics. Arch Ophthalmol 1987; 105: $1215-9$

7. Stephens RF, Shields JA. Diagnosis and management of cancer metastatic to the uvea. A study of 70 cases. Ophthalmology 1979; 86 : 1336-49.

8. Coleman DJ, Abramson DH, Jack RL, Franzen LA. Ultrasonic diagnosis of tumors of the choroid. Arch Ophthalmol 1974;91:344-54.

\section{MANIFESTACIONES INTRAOCULARES}

\section{DEL CÁNCER SISTÉMICO}

Resumen. El cáncer sistémico puede presentar manifestaciones intraoculares bajo tres aspectos: como manifestación de los tumores linfoproliferativos, como manifestación de tumores sólidos y en forma de síndrome paraneoplásico. [REV NEUROL 2000; 31. 1259-61] [http://www.revneurol.com/3112/j121259.pdf]

Palabras clave. Cáncer sistémico. Manifestaciones intraoculares. Tumores linfoproliferativos. Síndrome paraneoplásico.
9. Augsburger JJ. Fine needle aspiration biopsy of suspected metastatic cancers to the posterior uvea. Trans Am Ophthalmol Soc 1988; 86: 499-560.

10. Shields JA, Shields CL. Metastasic tumors to the intraocular structures. In Shields JA, Shields CL, eds. Intraocular tumors: a text and atlas. Philadelphia: WB Saunders; 1992. p. 208-38.

11. Volpe NJ, Albert DM. Metastasis to the uvea. In Albert DM, Jakobiec FA, eds. Principles and practice of ophthalmology. Philadelphia: WB Saunders; 1994. p. 3260-70.

12. Gragoudas ES. Current treatment of metastasic choroidal tumors. Oncology 1989; 3: 103-10.

13. Thirkill CE, Roth AM, Keltner JL. Cancer-associated retinopathy. Arch Ophthalmol 1897; 105: 372-5.

14. Keltner JL, Thirkill CE, Tyler NK, Roth AM. Management and monitoring of cancer-associated retinopathy. Arch Ophthalmol 1992; 110: 48-53.

15. Barr CC, Zimmerman LE, Curtin VT, Font RL. Bilateral diffuse uveal tumors associated with systemic neoplasm: a recently recognized syndrome. Arch Ophthalmol 1982; 100: 249-55.

\section{MANIFESTAÇÕES INTRAOCULARES \\ DO CANCRO SISTÉMICO}

Resumo. O cancro sistémioco pode apresentar manifestações intraoculares sob três aspectos: como manifestação dos tumores linfoproliferativos, como manifestação de tumores sólidos e sob forma de síndroma paraneoplásico. [REV NEUROL 2000; 31: 1259-61] [http:/ /www.revneurol.com/3112/j121259.pdf]

Palavras chave. Cancro sistémico. Maifestações intraoculares. Tumores linfoproliferativos. Síndroma paraneoplásico. 